\title{
Correction: Proportionality between variances in gene expression induced by noise and mutation: consequence of evolutionary robustness
}

\author{
Kunihiko Kaneko
}

Although the simulation data as well as the conclusion on the proportionality between $V_{i p}(i)$ and $V_{g}(i)$ in the work [1] is correct, interpretation of some data therein should be corrected. As the sampling number $(L=200)$ to measure the average gene expression level is not large enough, there is a bias in the estimate in $V_{g}(i)$. Finiteness in the number of sampling $L$ will generally cause a bias of the order of $V_{i p}(i) / L$, in the estimate of the variance $V_{g}(i)$. The too good proportionality between $V_{i p}(i)$ and $V_{g}(i)$ for large $\sigma$, shown in Figure two (a)(b) of [1] (especially for small $V_{g}(i)$ ), is due to this artifact. Accordingly, the sharp peak at $\sim 1 / L=1 / 200$ in Figure three of [1] is due to this insufficiency by the sample number.

Still, the proportionality between the two variances $V_{i p}(i)$ and $V_{g}(i)$, albeit not so sharp, holds, as already observed in the region with larger $V_{g}(i)$ in [1]. We have simulated the model with a larger number of samples, i.e., $N=L=1000$. As is shown in Figure 1, the proportionality is well discernible, where the proportion coefficient $V_{g}(i) / V_{i p}(i)$ decreased with the increase in the noise level $\sigma$, which was already observed in the broad peak beyond $1 / L$ in Figure three of [1]. This broad peak beyond $1 / L$ in Figure three of [1] was found to be sharper as $N$ was increased, from 200 to 1000 . This peak indeed corresponds to the proportion coefficient extracted from Figure 1 in the present Correction. As the noise level $\sigma$ was increased, the peak position $\rho=V_{g}(i) / V_{i p}(i)$ decreased. Hence for larger $\sigma$, larger $L$ is needed to get reliable estimate in the proportion coefficient. As for Figure five and Figure six of [1], the sharp proportionality for $V_{g}(i) \lesssim 0.001$ is due to the above bias, while the discussion therein concerns with the approach of $V_{g}(i)$ to $V_{i p}(i)$ at larger $V_{g}(i)$, which is not affected by the bias here.

Correspondence: kaneko@complex.c.u-tokyo.ac.jp

Department of Basic Science, Univ. of Tokyo 3-8-1 Komaba, Meguro-ku, Tokyo 153-8902, Japan
To sum up, the main claim of [1], i.e., proportionality between $V_{i p}(i)$ and $V_{g}(i)$ is valid, but the value of the proportion coefficient $\rho=V_{g}(i) / V_{i p}(i)$ should be corrected. It decreases with the noise level, in contrast to the discussion in [1] for large $\sigma$. Major factor on this proportionality is attributed to the correlation of each variance with the average value $\overline{\operatorname{Sign}(x(i))}$ : In other words, a state with an intermediate expression level (i.e., smaller $|\overline{\operatorname{Sign}(x(i))}|)$ can be more easily switched on or off, both by noise and also by mutation, and hence the variances generally increase as $|\overline{\operatorname{Sign}(x(i))}|$ approaches 0 . Still, some

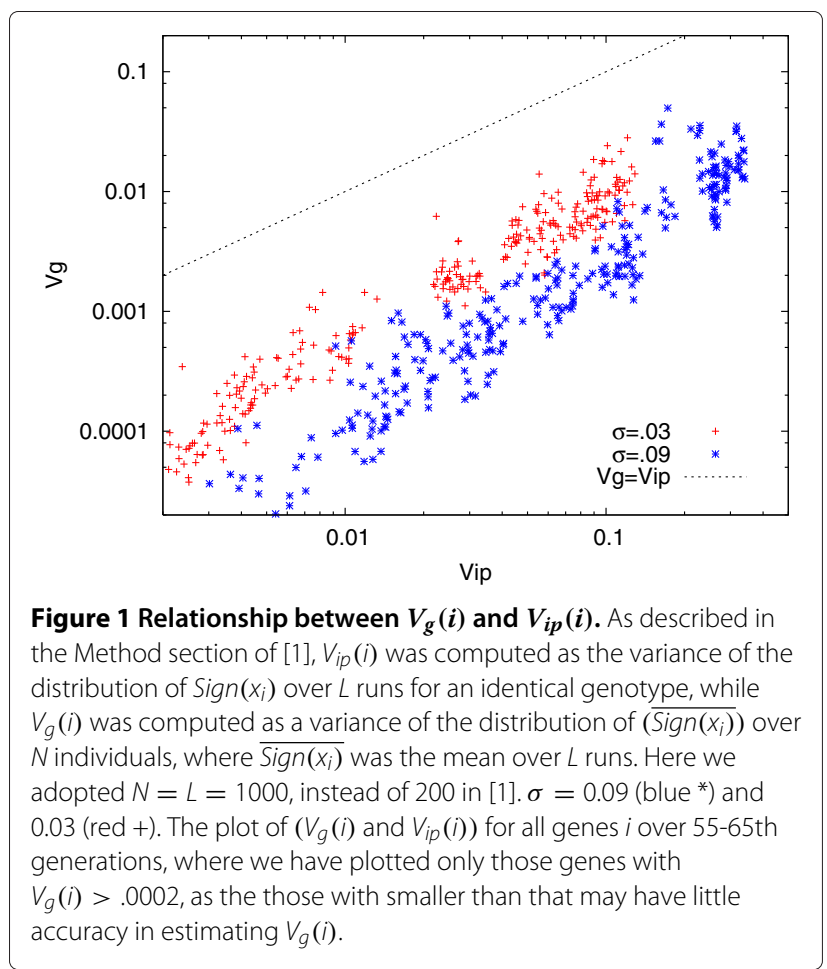


correlation between $V_{i p}(i)$ and $V_{g}(i)$ remains even after removing this correlation through $\overline{\operatorname{Sign}(x(i))}$.

I regret any inconvenience that misintepretation of the data with an insufficient sample size may have caused.

Received: 24 May 2012 Accepted: 3 December 2012

Published: 9 December 2012

\section{References}

1. Kaneko K: Proportionality between variances in gene expression induced by noise and mutation: consequence of evolutionary robustness. BMC Evol Biol 2011, 11:27.

doi:10.1186/1471-2148-12-240

Cite this article as: Kaneko: Correction: Proportionality between variances in gene expression induced by noise and mutation: consequence of evolutionary robustness. BMC Evolutionary Biology 2012 12:240.

Submit your next manuscript to BioMed Central and take full advantage of:

- Convenient online submission

- Thorough peer review

- No space constraints or color figure charges

- Immediate publication on acceptance

- Inclusion in PubMed, CAS, Scopus and Google Scholar

- Research which is freely available for redistribution

Submit your manuscript at www.biomedcentral.com/submit

\section{O) Biomed Central}

